\title{
THE TWO-CATHETER TECHNIQUE IN INDICATOR-DILUTION CARDIOVASCULAR STUDIES
}

\author{
BY \\ E. GREY DIMOND, ALBERTO BENCHIMOL, AND ERNEST W. CROW \\ From The Institute for Cardio-pulmonary Diseases, Scripps Clinic and Research Foundation, \\ La Jolla, California, U.S.A.
}

Received March 2, 1961

Indicator dilution technique is more widely used during cardiac catheterization for the diagnosis and rough estimate of the size of intracardiac shunts and valvular regurgitation (Korner and Shillingford, 1955; Fox and Wood, 1957; Swan and Wood, 1957; Woodward et al., 1957; Shillingford, 1958; Carter et al., 1959; Carleton et al., 1960; Jose et al., 1960).

Evans blue dye (T-1824) has long been used to record dye dilution curves, being of special value in the diagnosis of right-to-left shunts (Falholt and Fabricius, 1958). However, this dye has the disadvantage of accumulation in human tissue and is influenced by the blood oxygen saturation; therefore, it cannot be used to record curves with venous blood.

With the development of a new dye by Fox and Wood (1957) (tricarbocyanine dye II-cardiogreen)* it became possible to record indicator dilution curves from the right heart or venous circulation since this dye is not influenced by oxygen saturation of the blood. In the present study, we intend to report the usefulness of the dye dilution curve from the right side of the heart, using a two-catheter technique in patients with valvular regurgitation and left-to-right shunt in whom the blood oxygen saturation did not reveal an intracardiac shunt or adequately did not locate it.

The dye dilution curve with injection into the right heart and sampling at the systemic artery does not provide information regarding location of a left-to-right shunt. It may but suggest the presence of a shunt; and in many cases it does not differentiate a curve due to valvular regurgitation from a curve of a patient with left-to-right shunt. In both circumstances, there is a prolonged disappearance curve with absence of a small recirculation curve. Thus, in order to make the indicator dilution technique useful in the diagnosis of a left-to-right shunt, injections and sampling sites have to be in the right heart.

\section{METHODS}

Indicator dilution curves from the right side of the heart were recorded in sixty-two patients with congenital heart disease and in nineteen with acquired heart disease. The patients varied from one month to fifty-five years of age.

In order to identify and localize the presence of an intracardiac shunt or valvular regurgitation with this method, it is necessary to use two catheters at different locations for injection and sampling. It is possible to use a double lumen catheter, with injection being made through the distal lumen and sampling at the proximal lumen. When a two-catheter technique was used, a No. 6 or No. 7

This study was supported in part by funds from The National Heart Institute Graduate Training Program \#HTS5210; The National Heart Institute Research Grant \#H-5575; and the Timkin-Sturges Cardio-Vascular Research Grant.

* Hynson, Westcott and Dunning, Inc.

$2-\mathrm{S}$ 
angiography catheter was preferred (Fig. 1). The angiography catheters were used instead of a single opening catheter because with the latter, during withdrawal, the catheter tip may rest flush with the heart wall and the blood flow consequently may be obstructed.

All patients were studied by right heart catheterization and a great majority by selective cineangiocardiography. The diagnosis was confirmed by operation in most cases and occasionally by postmortem examination. In 23 patients, the blood oxygen saturation was not diagnostic of a left-toright shunt or the origin of the shunt was not precisely located. This group of patients is the main object of the study. The oxygen saturation and content of blood samples taken during cardiac catheterization were determined by Brinkman-Zigstra hæmoreflector and the Van Slyke and McNeill method (1924). For this special study, a large number of blood samples were taken from each cardiac chamber or vessel in a very rapid sequence. One catheter was first positioned in the pulmonary artery and the other catheter in the right ventricle and samples taken from there to the vena cava; simultaneous samples were taken from two different locations. The total time spent in the collection of the blood samples varied from fifteen to thirty minutes with an average of twenty-two minutes. A great number of samples were taken intentionally and the average per patient was twenty-two samples from the following locations: pulmonary artery (main, left, and right, two of each); right ventricle (inflow, mid, apex, and outflow, two of each); right atrium (inflow, mid, outflow, two of each); superior and inferior vena cava, two of each; systemic artery, two. Pressure measurements were performed with a P 23 D Statham strain gauge with a Sanborn polyviso direct writer recorder. The cardiac output was determined using the Fick principle. Calculation of the flows, shunts, and areas were made according to the method of Gorlin and Gorlin (1951).

Indicator dilution studies were performed during cardiac catheterization after the patient had been given mild sedation. Tricarbocyanine (Fox and Wood, 1960) was used in all cases. In the beginning of our study, the Waters densitometer (XC 100A) was employed and the curves recorded by a Sanborn poly-viso direct writer recorder at a speed of $5.0 \mathrm{~mm}$. a second. The blood was withdrawn with a pump at a constant rate of $30.0 \mathrm{ml}$. a minute. At the present time, we are using a Colson cuvette densitometer (No. 103-IR) and the curves recorded on a photographic paper (Electronics for medicine DR-8). The amount of dye used per injection varied according to the body weight, but an average of $5.0 \mathrm{mg}$. per injection was used in most patients. Several injections were made in individual cases but an arbitrary total dose calculated to be $2.0 \mathrm{mg}$. $/ \mathrm{kg}$. of body weight was not exceeded. At the present time, we have been using a maximal dose, $5.0 \mathrm{mg} . / \mathrm{kg}$. of body weight (Fox and Wood, 1960) without untoward side effects. An average of six injections per patient was sufficient.

For the diagnosis of an aorto-pulmonary communication, the injection was made at the right pulmonary artery and sampling at the left pulmonary artery. In case of ventricular septal defect, injection was made at the main pulmonary artery with sampling at the right ventricle (midportion of outflow tract (Fig. 1)). In case of atrial septal defect, injections were made at the right ventricle and samplings at the mid right atrium. In case of anomalous pulmonary venous drainage, injection was made at the right or left pulmonary artery and sampling at the superior or inferior vena cava. Since the appearance time varies from patient to patient, it was necessary to record a curve proximal and distal to the left-to-right shunt in every case.

At first we made no attempt to measure the size of the shunts by the dye curves. Now the curve is calibrated using the dilution method of Nicholson and Wood (1951) and the calculations made with the technique of Wood et al. (1957) and Hetzel et al. (1959). All curves illustrated in this report were obtained from patients whose diagnoses were proved by operation.

\section{RESULTS}

Normal Curve from the Right Heart. The normal dye dilution curve from the right heart is demonstrated (Fig. 2). The curve shows a somewhat long appearance time, low-peak concentration and small or no recirculation curve. This is due to the fact that the bolus of dye injected into the 


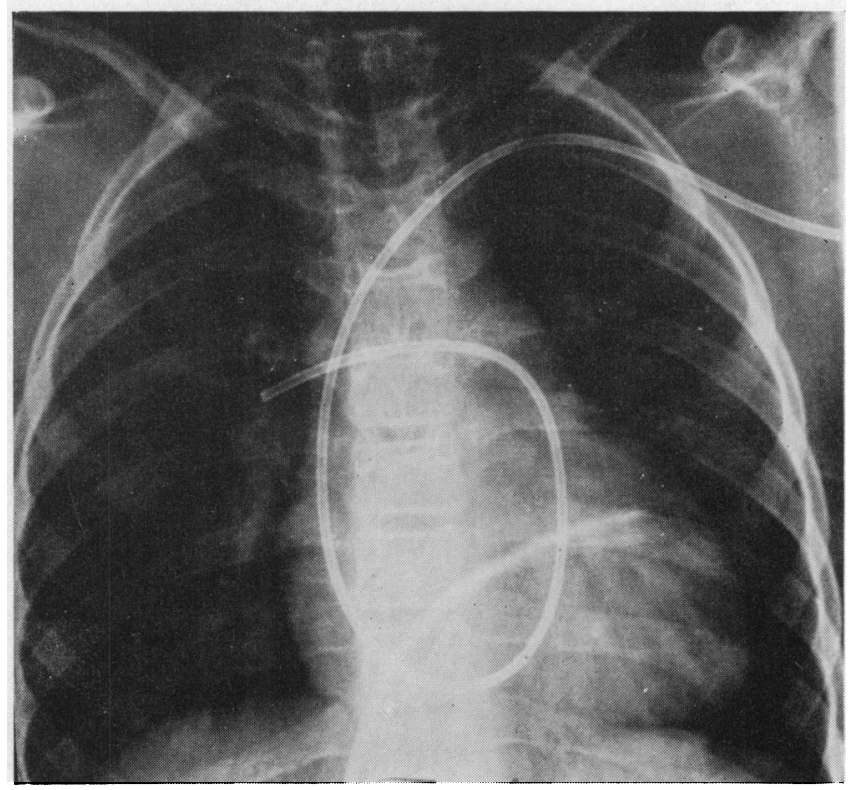

Fig. 1.-Chest X-ray showing the location of the two catheters. The catheter from the inferior vena cava is at the outflow tract of the right ventricle and the catheter from the superior vena cava is in the right pulmonary artery.
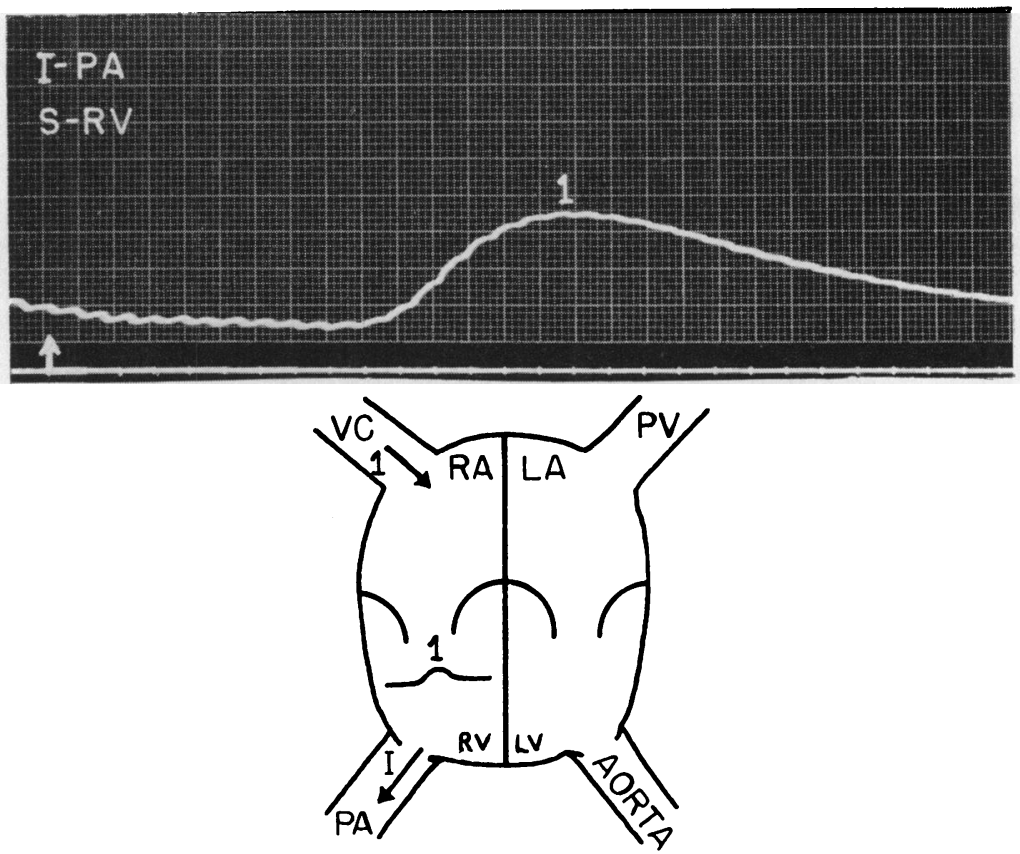

FIG. 2.-Normal curve (marked 1). Note the long appearance time, slow build-up time and disappearance time. The time of injection is marked with an arrow. The time mark at the bottom of the curves in all illustrations represents one second. $\mathrm{VC}=$ Vena cava. $R A=$ Right atrium. $R V=$ Right ventricle. $\mathbf{P A}=$ Pulmonary artery. $\mathbf{P V}=$ Pulmonary vein. $\mathrm{LA}=$ Left atrium. $\mathrm{LV}=$ Left ventricle. $I=$ Site of injection of dye. $\mathrm{S}=$ Site of sampling of dye. 
pulmonary artery, right ventricle, or right atrium has to cross the pulmonary and systemic circulations and a considerable amount of dilution takes place through numerous anatomical pathways.

Left-to-Right Shunt. Aorto-pulmonary Communication. In this condition, the dye was injected into the right pulmonary artery and the sampling site was the left pulmonary artery. In the case of patent ductus, the streaming effect of the left-to-right shunt to the left pulmonary artery strongly emphasizes the need to have the left and not the right pulmonary artery as a sampling site. The curve in this condition shows an abnormal early appearance time just preceding the main venous curve (Fig. 3).

Ventricular and Atrial Septal Defect. In cases of ventricular septal defect, the injection was made at the pulmonary artery and the sampling at the mid or outflow tract of the right ventricle. When the catheter tip is placed at the apex, it is possible to fail to record an early appearance time due to the streaming effect of the left-to-right shunt which is directed to the mid or outflow tract of the right ventricle. In cases of atrial septal defect, injection was made at the pulmonary artery or right ventricle and the sampling at the mid-right atrium (Fig. 4, 5, and 6). In both conditions, the curves characteristically revealed an early appearance time preceding the main venous curves (Fig. 7). The association of anomalous venous drainage with atrial septal defect can be easily recognized with this technique (Fig. 8).

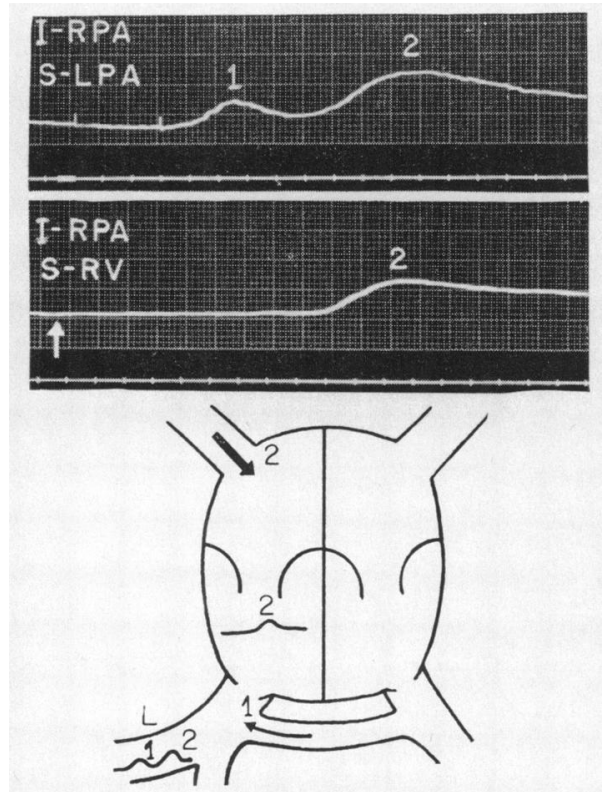

Fig. 3.-Patent ductus arteriosus with left-toright shunt and normal pulmonary artery pressure. Note the early appearance curve in the upper tracing with sampling at the left pulmonary artery. Normal right ventricular curve. No. 1 represents the early appearance curve of the left-to-right shunt, and No. 2 the main venous curve. RPA = Right pulmonary artery. LPA = Left pulmonary artery. $R V=$ Right ventricle. I=Site of injection of dye. $\mathrm{S}=$ Site of sampling of dye.

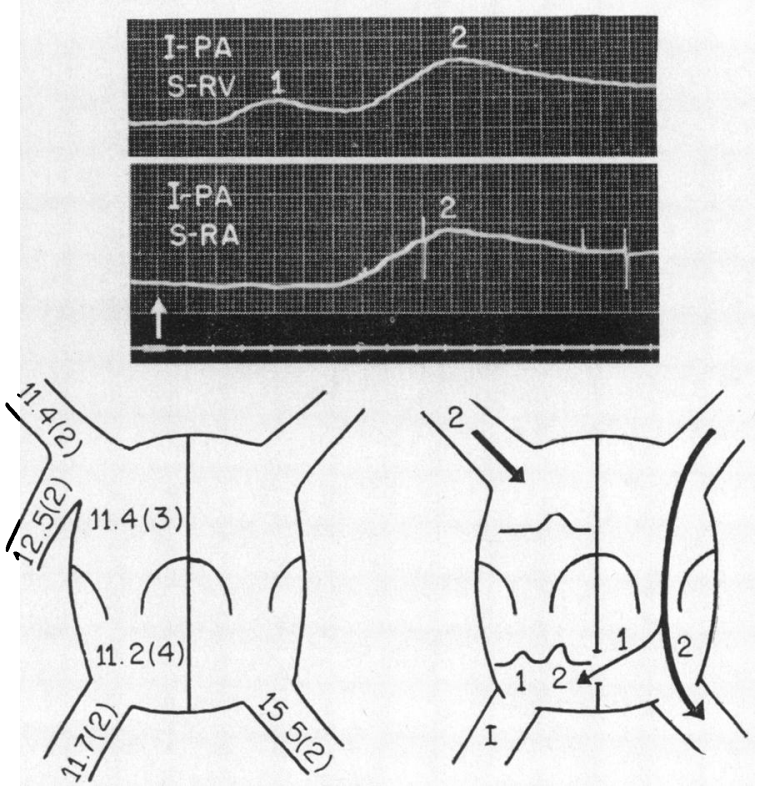

FIG. 4.-Ventricular septal defect with left-to-right shunt and normal pulmonary artery pressure. Note that the oxygen content data does not reveal a left-to-right shunt. A total of fifteen blood samples were taken from the right heart in this patient. The dye dilution curve from the right ventricle is diagnostic of a left-to-right shunt at this level. Observe the early curve immediately preceding the main curve. Compare with Fig. 3, 5, 6, and 7 . The numbers in the left-hand diagram represent the values of the oxygen content in volumes per cent. The numbers in parentheses represent the total number of samples taken from the specific chamber or vessel. $\mathbf{P A}=$ Pulmonary artery. $\mathbf{R V}=$ Right ventricle. $\mathbf{R A}=$ Right atrium. I=Site of injection of dye. $S=$ Site of sampling of dye. No. 1 represents the early appearance curve of the left-to-right shunt, and No. 2 the main venous curve. 

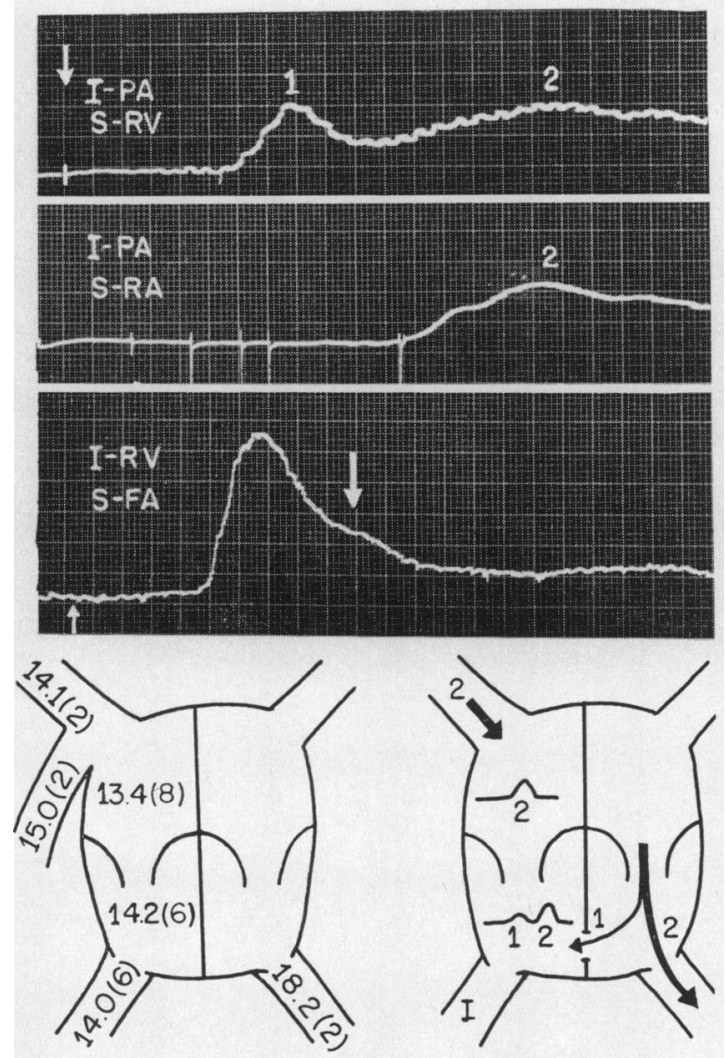

Fig. 5.-Ventricular septal defect with left-to-right shunt and mild pulmonary hypertension. The blood oxygen content of the right ventricle reveals an increase of $0.8 \mathrm{vol}$. per cent, and the pulmonary artery $0.6 \mathrm{vol}$. per cent, above that in the right atrium. The dye curve, with sampling at the right ventricle, immediately identifies the presence of a left-to-right shunt by an early appearance time. The right atrial curve is normal. Observe the interruption of the descending limb of the femoral artery curve (arrowed) reflecting the shunted blood. PA $=$ Pulmonary artery. $\mathrm{RV}=$ Right ventricle. $\mathrm{RA}=\mathrm{Right}$ atrium. $\mathrm{FA}=$ Femoral artery. I = Site of injection of dye. $S=$ Site of sampling of dye. No. 1 represents the early appearance curve of the left-to-right shunt, and No. 2 the main venous curve. The numbers in the lefthand diagram represent the values of the oxygen content in volumes per cent. The numbers in parenthesis represent the total number of samples taken.
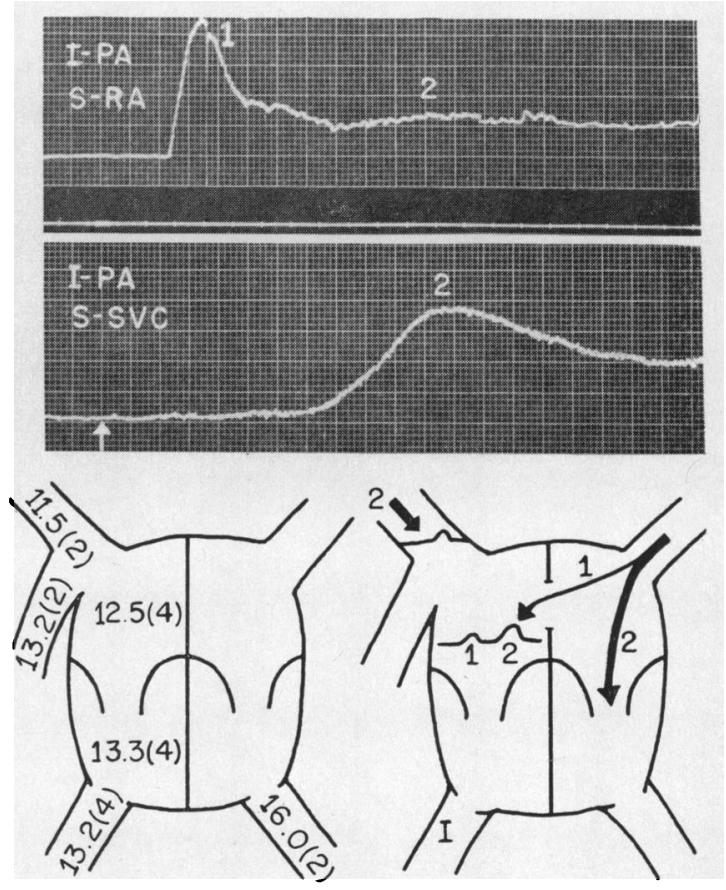

FIG. 6.-Atrial septal defect with left-to-right shunt and normal pulmonary artery pressure. The blood oxygen content was misleading, revealing an increase of 0.8 vol. per cent at the ventricular level suggesting a left-to-right shunt at this level and none at the atrial level. The dye curve, with a sampling at the right atrium, revealed a large early appearance curve typical of left-to-right shunt. The curve recorded from the superior vena cava is normal thus localizing the shunt at atrial level. $\mathbf{P A}=$ Pulmonary artery. $\mathrm{RA}=\mathrm{Right}$ atrium. $\mathrm{SVC}=$ Superior vena cava. I=Site of injection of dye. S=Site of sampling of dye. No. 1 represents the early appearance curve of the left-to-right shunt, and No. 2 the main venous curve. The numbers in the left-hand diagram represent the values of the oxygen content in volumes per cent. The numbers in parenthesis represent the total number of samples taken.

In the majority of cases with atrial septal defect, it is possible to enter the left atrium through the defect. In this circumstance, injection made in the left atrium with sampling at the right atrium will identify a left-to-right shunt. In some infants in whom the foramen ovale is not completely closed, manœuvring the catheter against the septum may enable one to get into the left atrium. The two catheter method is then especially helpful to differentiate a true septal defect from a patent foramen ovale, since in the latter, no early appearance time will be recorded.

Anomalous Venous Return. This method is especially useful in the diagnosis of an anomalous pulmonary venous drainage to the right atrium, superior vena cava, or inferior vena cava. In the 

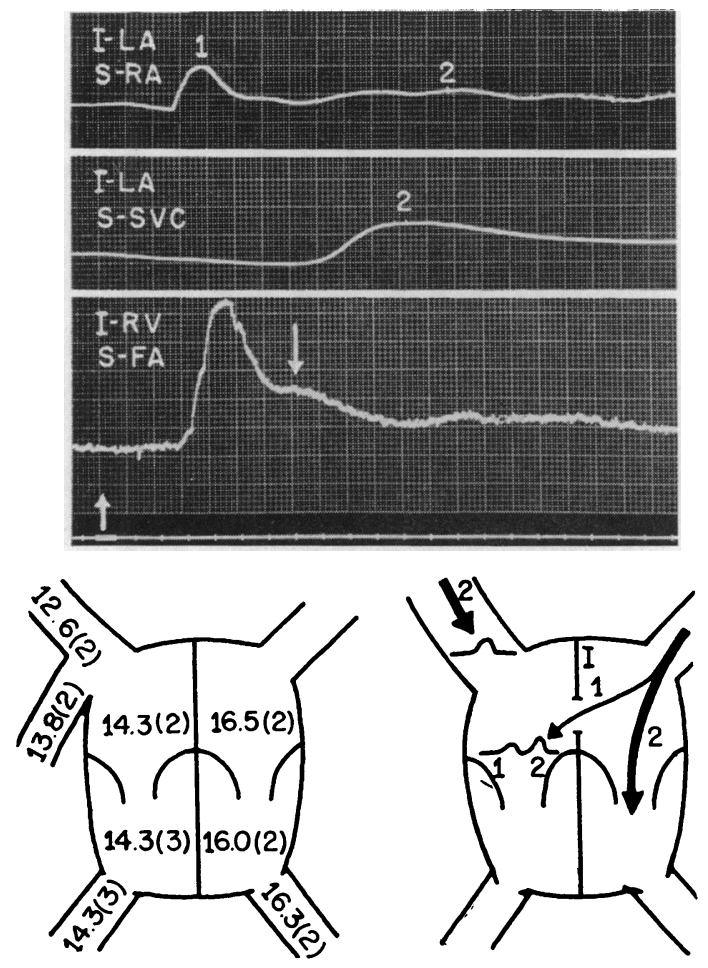

FIG. 7.-Atrial septal defect, secundum type, with the left-to-right shunt and normal pulmonary artery pressure. The blood oxygen content revealed a very small equivocal rise at the atrial level $(0.5$ vol. $\%$ ). The dye curve with injection at the left atrium and sampling at the right atrium and superior vena cava revealed an early appearance curve at the right atrium and none at the superior vena thus localizing the shunt at the atrial level. Observe the interruption of the descending limb of the femoral artery curve (arrowed) reflecting the shunted blood. $\mathrm{LA}=$ Left atrium. $\mathrm{RA}=\mathrm{Right}$ atrium. $\mathrm{SVC}=$ Superior vena cava. $R V=$ Right ventricle. $F A=$ Femoral artery. $I=$ Site of injection of dye. $S=$ Site of sampling of dye. No. 1 represents the early appearance curve of the left-to-right shunt and No. 2 the main venous curve. The numbers in the left-hand diagram represent the values of the oxygen content in volumes per cent. The numbers in parenthesis represent the numbers of samples taken.
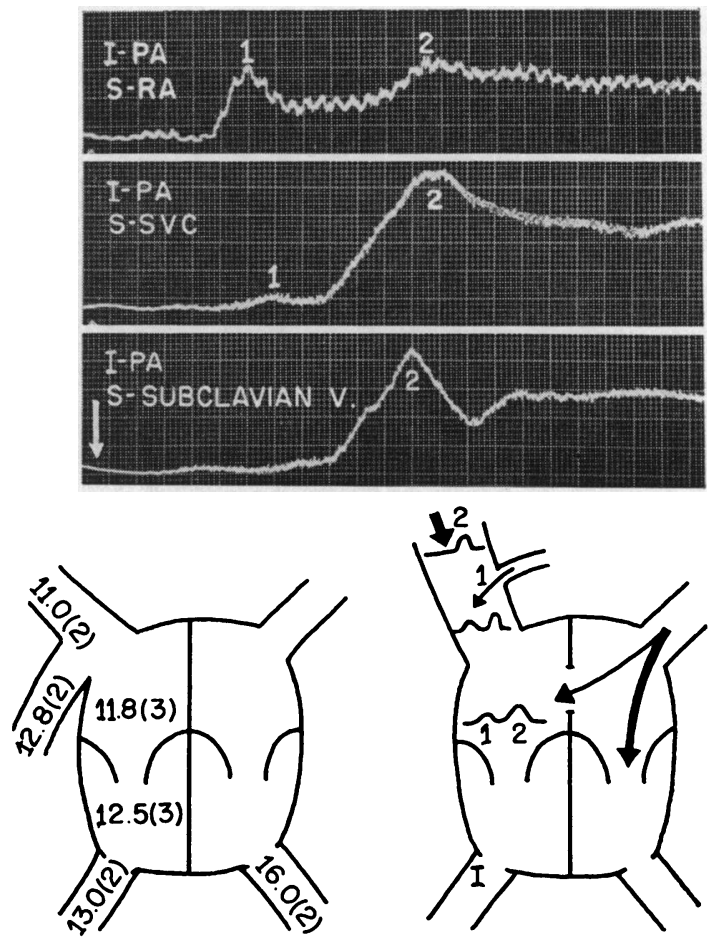

FIG. 8.-Atrial septal defect with anomalous venous drainage at the superior vena cava. The blood oxygen content was misleading revealing a small $(0.5 \mathrm{vol} . \%)$ rise at the pulmonary artery level and no rise at the right atrium. The right atrial dye curve revealed an early appearance time typical of a left-to-right shunt at this level. In addition, the curve from the superior vena cava also revealed an early appearance curve which was not present at the subclavian vein level thus localizing an additional shunt at the superior vena cava. $\mathbf{P A}=$ Pulmonary artery. $\mathrm{RA}=\mathrm{Right}$ atrium. $\mathrm{SVC}=$ Superior vena cava. $V=$ Vein. $I=$ Site of injection of dye. $S=$ Site of sampling of dye. No. 1 represents the early appearance curve of the left-to-right shunt and No. 2 the main venous curve. The numbers in the left-hand diagram represent the values of the oxygen content in volumes per cent. The numbers in parenthesis represent the number of samples taken.

majority of cases, it is not only possible to identify the presence of an anomalous vein but also to determine the lung of origin. If one recognizes the presence of an early appearance time at the superior or inferior vena cava or right atrium, injection of tricarbocyanine should then be made at the right and left pulmonary arteries, respectively. The failure to record an early appearance time from one lung will immediately identify the other lung as the point of origin of the anomalous drainage (Fig. 9). It is also possible to determine the lobar origin of a pulmonary vein using the same technique as described above. In this particular situation, the dye is injected into each branch of the pulmonary artery and the sampling site at the point of drainage of the anomalous vein.

Valvular Regurgitation. If an injection is made distal to an incompetent valve and the sampling site is proximal, an early appearance time will be recorded with regurgitation of dye into the sampled 

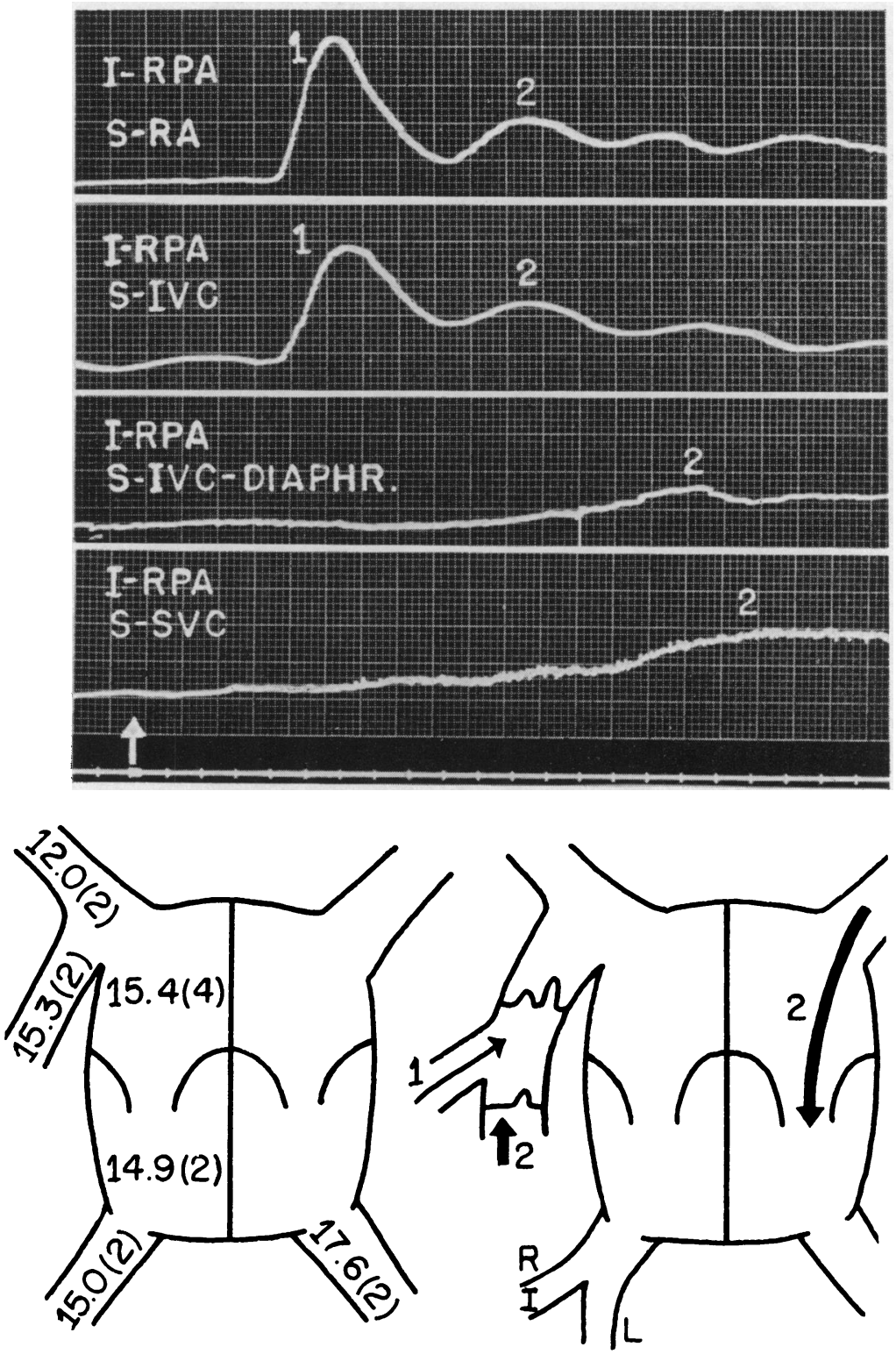

FIG. 9.-Total anomalous pulmonary venous drainage of the right lung to the inferior vena cava. The blood oxygen content study revealed high values at the inferior vena cava. The dye curve with injection at the right pulmonary artery and sampling at the right atrium and inferior vena cava above the diaphragm revealed an early appearance curve. The curve recorded one inch below the diaphragm revealed a normal venous curve thus localizing the left-to-right shunt at the level of the diaphragm. As the injection was made in the right pulmonary artery, the origin of the anomalous vein was identified as being the right lung. RPA=Right pulmonary artery. $R A=R$ ight atrium. IVC=Inferior vena cava. Diaph.=Diaphragm. SVC=Superior vena cava. $I=$ Site of injection of dye. $S=$ Site of sampling of dye. No. 1 represents the early appearance curve of the left-to-right shunt and No. 2 the main venous curve. The numbers in the left-hand diagram represent the values of the oxygen content in volume per cent. The numbers in parenthesis represent the number of samples taken. 
chamber. In this fashion, pulmonary, tricuspid, mitral, or aortic regurgitation is readily recognized as shown in Fig. 10 and 11.

The recognition by this technique of either valvular regurgitation or left-to-right shunt is based of the presence of an early appearance of dye at the sampling site. However, it must be pointed out that an early curve of valvular regurgitation occurs well before the main venous curve in contradistinction to curves diagnostic of a left-to-right shunt in which the early appearance curve just
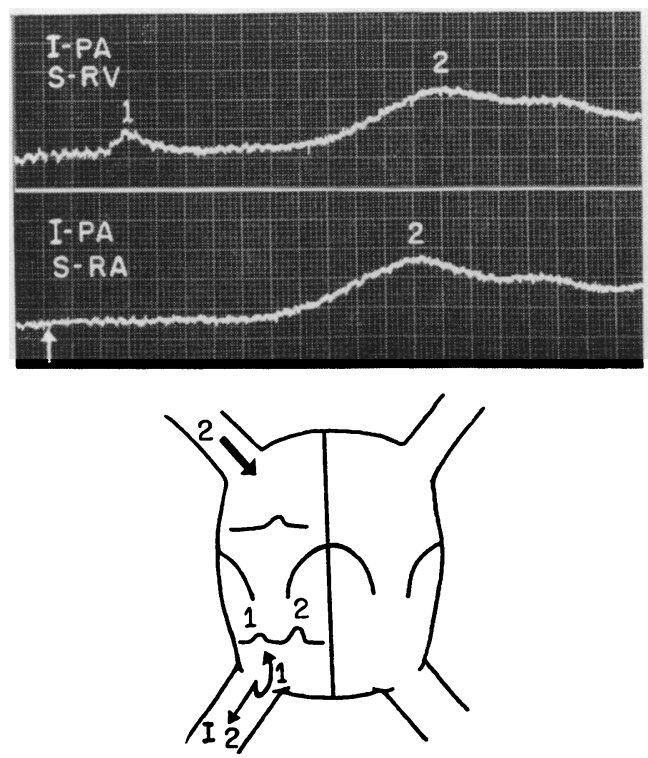

Fig. 10.-Pulmonary regurgitation. Blood oxygen content did not reveal a left-to-right shunt. The dye curve with injection at the main pulmonary artery with sampling at the right ventricle revealed an early appearance time consistent with pulmonary regurgitation. The early curve (1) occurs eight seconds before the main curve (2), differentiating regurgitant from leftto-right shunt curve. See text. Compare with Fig. 3, 5, 6, and 7. $\mathrm{PA}=$ Pulmonary artery. $\mathbf{R V}=$ Right ventricle. $\mathbf{R A}=$ Right atrium. I $=$ Site of injection of dye. $\mathrm{S}=$ Site of sampling of dye.

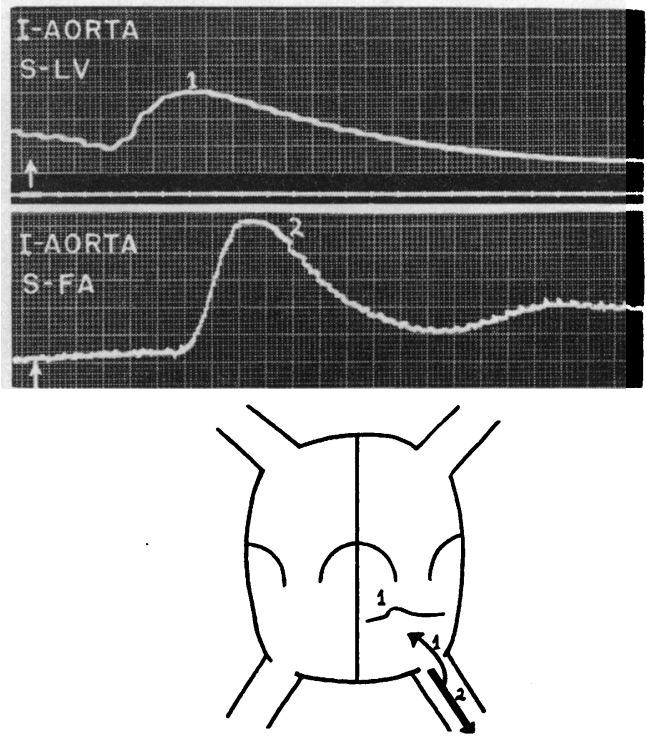

FIG. 11.-Aortic regurgitation. Note the large, early appearance curve (1), when injection was made at the ascending aorta and sampling at the left ventricle. Observe normal curve (2), when the injection was made at the descending aorta with sampling at the femoral artery. Compare with Fig. 10. $L V=L e f t$ ventricle. FA=Femoral artery. I=Site of injection of dye. $S=$ Site of sampling of dye.

precedes the main venous curve. We have found no difficulty in differentiating a regurgitant curve from the curve of a left-to-right shunt (Compare Fig. 3, 4, 5, 6, 7, and 9 with Fig. 10 and 11).

When combined catheterization of the right and left heart is performed, the dye dilution twocatheter technique becomes a valuable and precise means of localizing intracardiac shunts and valvular regurgitation. It is especially useful in the presence of an endocardial cushion defect when the possibility of valvular regurgitation due to a cleft of the atrio-ventricular valve associated with left-to-right shunt is contemplated (Fig. 12). The identification of a left ventricular to right atrial shunt is made possible with this technique.

\section{Discussion}

Indicator dilution curves with tricarbocyanine, when recorded from the right side of the heart, provide a very accurate way to localize a left-to-right shunt (Swan and Wood, 1957; Falholt and Fabricius, 1958; Oakley et al., 1960; Sanders and Morrow, 1959). With this technique, it is possible to detect a left-to-right shunt of a magnitude of 20 per cent (Fox and Wood, 1960). It 

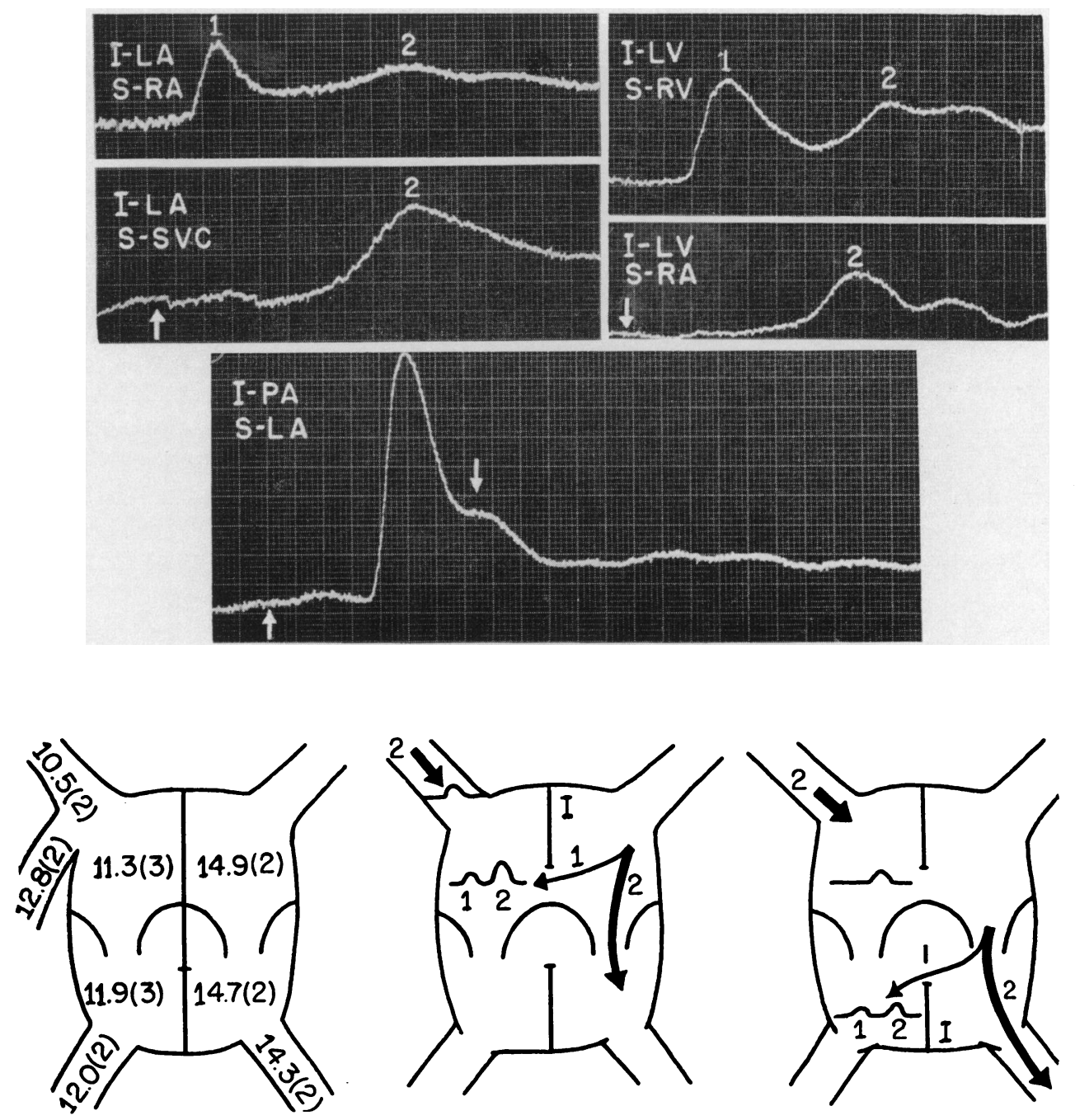

FIG. 12.-Endocardial cushion defect with left-to-right shunt and minimal pulmonary hypertension. The oxygen values throughout the right heart did not reveal a left-to-right shunt. All four chambers of the heart were entered during catheterization. The dye curve, with injection of the left atrium and sampling at the right atrium, identified a left-to-right shunt at the atrial level. In addition, the injection made at the left ventricle with sampling at the right ventricle was diagnostic of an additional arteriovenous shunt at the ventricular level. $L A=$ Left atrium. RA=Right atrium. SVC $=$ Superior vena cava. $L V=$ Left ventricle. $\quad R V=$ Right ventricle. $\quad P A=P u l m o n a r y$ artery. $I=$ Site of injection of dye. $S=$ Site of sampling of dye. No. 1 represents the early appearance curve of the left-to-right shunt and No. 2 the main venous curve. The numbers in the left-hand diagram represent the values of the oxygen content in volumes per cent. The numbers in parenthesis represent the number of samples taken.

is agreed that measurement of change in $\mathrm{O}_{2}$ saturation of the venous blood is a somewhat insensitive means of localizing and estimating the size of a left-to-right shunt (Fig. 4, 5, 6, 7, 8, and 9). Changes in the metabolic rate and oxygen consumption, streaming effect, etc. makes this method far from being ideal (Fox et al., 1957). This is especially true with children in whom it is very difficult to maintain a steady state during cardiac catheterization and in whom the oxygen values vary rapidly and markedly. Furthermore, determination of cardiac output becomes a 


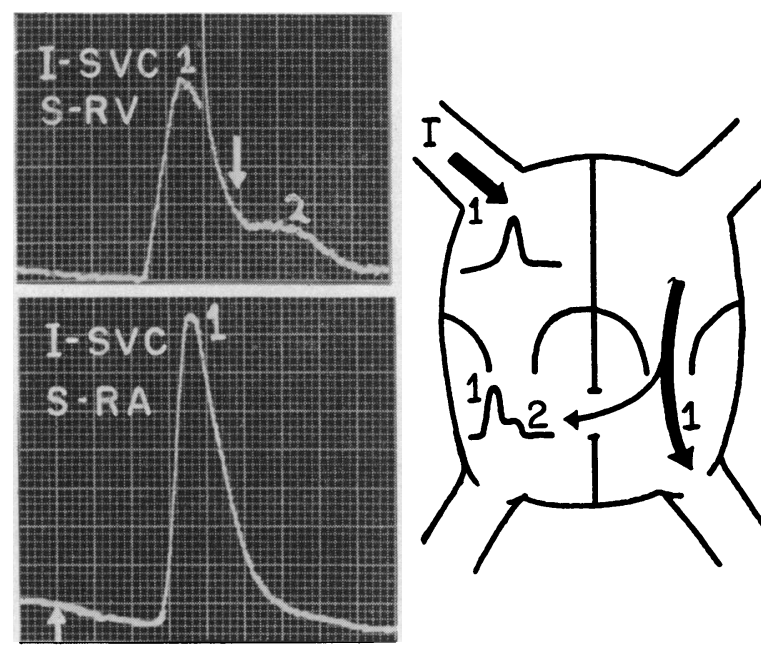

FIG. 13.-Ventricular septal defect with left-to-right shunt and normal pulmonary artery pressure. The injections were made on the superior vena cava with sampling at the right ventricle and right atrium. Note the interruption (2) of the descending limb in the right ventricular, but not the atrial curve, thus localizing the shunt to ventricular level. SVC=Superior vena cava. $R V=$ Right ventricle. $R A=R$ ight atrium. I=Site of injection of dye. $S=$ Site of sampling of dye. No. 1 represents the early appearance time of the main venous curve. problem in a child below the age of eight due to difficulties in collecting expired air for the determination of oxygen consumption. Dye dilution curves, in addition to other advantages mentioned, provide a simple way to determine output in children above the age of two years.

In addition, the right side dye curve with the two-catheter technique provides the only way to identify precisely the lung and lobe of origin of an anomalous pulmonary vein.

Recently, Braunwald et al. (1959) suggested a simple method to detect a left-to-right shunt using a peripheral vein as the injection site and sampling at the pulmonary artery, right ventricle, right atrium, and vena cava. The venous curve, with this technique, will reveal an interruption in the descending limb in the chamber distal to the left-to-right shunt (Fig. 13 and 14). We have used this techniqe occasionally in small children with good results. However, it is not as reliable as the two-catheter technique. In many instances, the curves are quite difficult to interpret. The inter-

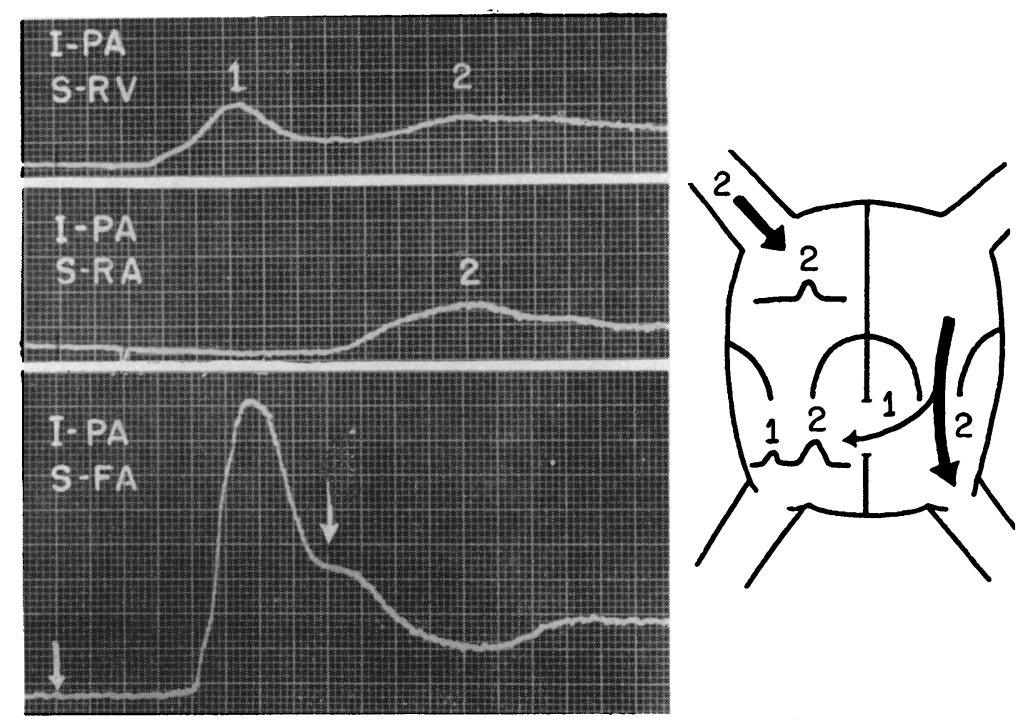

Fig. 14.-Same patient as in Fig. 13. Note the early appearance curve (1) at the right ventricle typical of a left-to-right shunt, and the normal venous curve (2) in the right atrium. The oxygen values in this patient revealed a rise at the pulmonary artery level and none at the right ventricle despite the fact that eight samples were taken from this chamber. PA=Pulmonary artery. $R V=$ Right ventricle. $R A=$ Right atrium. $F A=$ Femoral artery. $\mathrm{I}=$ Site of injection of dye. $\mathrm{S}=$ Site of sampling of dye. Observe the interruption of the descending limb of the femoral artery curve (arrowed) reflecting the shunted blood. 
ruption of the decending limb is not always distinct and the interpretation of the curve therefore difficult. In addition, it does not provide an accurate way to locate the origin of an anomalous venous drainage.

This two-catheter technique can also be successfully used during left heart catheterization to identify mitral and aortic regurgitation (Fig. 11). One possible disadvantage of the two-catheter technique is the necessity for multiple catheters in the right side of the heart. However, one may use a double lumen catheter or place two catheters in the same vein. In adults, it is usually possible to use the medial antecubital vein for one catheter and one small branch of the same vein for the second catheter. Lately, in children below the age of four, we have been using successfully the superficial femoral vein below the junction with the saphenous vein and making two separate incisions. This is done in order to secure better hemostasis, which is more difficult to accomplish if two catheters are inserted in the same incision.

\section{SUMMARY}

A study of the indicator dilution curve as recorded from the right side of the heart is presented. Sixty-two patients were studied with the two-catheter technique. Injection of the indicator with this technique is made distal to the shunt and the sampling site immediately proximal to the left-to-right shunt.

In all cases it was possible to localize accurately a left-to-right shunt based on the early appearance curve just preceding the main venous curve.

This method is also useful in detecting the presence of valvular regurgitation. In the presence of regurgitation, the early appearance curve precedes the main venous curve by several seconds making the differentiation between regurgitant and left-to-right shunt curves easily available.

This technique is especially useful to locate precisely the origin of the anomalous pulmonary venous drainage. In most cases, it is possible to identify its lobar origin.

A comparison of this method is made with the blood oxygen content method of detecting a left-to-right shunt and the disadvantage of oxygen determination is emphasized.

We wish to thank Dr. Yen Shen and Miss Dorothy Thomas for their technical assistance and Mrs. Carol Dafoe and Mrs. Betty Albert for their secretarial assistance. Many of the patients studied were seen in the Cardiovascular Laboratory, University of Kansas Medical Center, Kansas City, Kansas. The cardiogreen dye was kindly supplied by Hynson, Westcott and Dunning, Inc.

\section{REFERENCES}

Braunwald, E., Pfaff, W. W., Long, R. T. L., and Morrow, A. G. (1959). Circulation, $20,875$.

Carleton, R. A., Levinson, G. E., and Abelman, W. H. (1960). Amer. Heart J., 60, 396.

Carter, S. A., Swan, H. J. C., and Wood, E. H. (1959). Circulation, 19, 430.

Falholt, W., and Fabricius, J. (1958). Brit. Heart J., 20, 117.

Fox, I. J., Brooker, L. G. S., Heseltine, D. W., Essex, H. E., and Wood, E. H. (1957). Proc. Mayo Clin., $32,478$.

, and Wood, E. H. (1957). Proc. Mayo Clin., 32, 541.

- (1960). Proc. Mayo Clin., 35, 732.

Gorlin, R., and Gorlin, S. G. (1951). Amer. Heart J., 41, 1.

Hetzel, P. S., Swan, H. J. C., and Wood, E. H. (1959). Intravascular Catheterization. p. 539. Charles C. Thomas, Springfield, Illinois.

Jose, A. D., McGaff, C. J., and Milnor, W. R. (1960). Amer. Heart J., 60, 408.

Korner, P. I., and Shillingford, J. P. (1955). Clin. Sci., 14, 553.

Nicholson, J. W., and Wood, E. H. (1951). J. Lab. clin. Med., 38, 588.

Oakley, C., Taylor, S., Wilcken, D., Hollman, A., Goodwin, J. F., and Shillingford, J. (1960). Brit. Heart J., 22, 533.

Sanders, R. J., and Morrow, A. G. (1959). Amer. J. Med., 26, 508.

Shillingford, J. P. (1958). Brit. Heart J., $20,229$.

Swan, H. J. C., and Wood, E. H. (1957). Proc. Mayo Clin., 32, 486 and 496.

Van Slyke, D. D., and McNeill, J. M. (1924). J. biol. Chem., 61, 323.

Wood, E. H., Swan, H. J. C., and Helmholz, H. F. (1957). Proc. Mayo Clin., 32, 464.

Woodward, E., Burchell, H. B., and Wood, E. H. (1957). Proc. Mayo Clin., 32, 518. 